Service social

\title{
La résilience chez les jeunes hébergés en milieu substitut
}

\section{Sylvie Drapeau, Marie-Christine Saint-Jacques, Rachel Lépine, Gilles Bégin et Martine Bernard}

Volume 51, numéro 1, 2004

URI : https://id.erudit.org/iderudit/012713ar

DOI : https://doi.org/10.7202/012713ar

Aller au sommaire du numéro

\section{Éditeur(s)}

École de service social de l'Université Laval

ISSN

1708-1734 (numérique)

Découvrir la revue

Citer cet article

Drapeau, S., Saint-Jacques, M.-C., Lépine, R., Bégin, G. \& Bernard, M. (2004). La résilience chez les jeunes hébergés en milieu substitut. Service social, 51(1),

78-97. https://doi.org/10.7202/012713ar

\section{Résumé de l'article}

L'objectif général de cette recherche qualitative est de mettre en lumière les facteurs de protection, mais aussi les facteurs de vulnérabilité, présents chez des adolescents hébergés en milieu substitut, considérés comme étant résilients. Douze jeunes (six garçons et six filles) âgés de 14 à 17 ans et placés pour des problèmes de comportement ont participé à cette étude. Ces jeunes, placés depuis en moyenne 5,6 ans, ont été identifiés comme étant résilients (à partir d'une grille soumise aux intervenants et d'une discussion clinique). Deux entrevues ont été menées avec chaque jeune. Les résultats mettent en évidence les facteurs de protection environnementaux (présence de liens significatifs avec des adultes, notamment des intervenants, services de qualité et sources de valorisation) et individuels (confiance en soi, perception de contrôle, stratégies d'adaptation) qui ont favorisé la résilience de ces jeunes. 


\title{
La résilience chez les jeunes hébergés en milieu substitut ${ }^{1}$
}

\author{
Sylvie DRAPEAU ${ }^{2}$ \\ Professeure titulaire \\ Dép. des fondements et pratiques en éducation \\ Université Laval \\ Marie-Christine SAINT-JACQUES ${ }^{2}$ \\ Professeure \\ École de service social \\ Université Laval \\ Rachel LEPINE ${ }^{2}$ \\ Professionnelle de recherche \\ Jefar \\ Université Laval \\ Gilles BEGIN \\ Centre jeunesse de Québec \\ Institut universitaire sur les jeunes en difficulté \\ Martine BERNARD \\ Centre jeunesse de Québec \\ Institut universitaire sur les jeunes en difficulté
}

L'objectif général de cette recherche qualitative est de mettre en lumière les facteurs de protection, mais aussi les facteurs de vulnérabilité, présents chez des adolescents hébergés en milieu substitut, considérés comme étant résilients. Douze jeunes (six garçons et six filles) âgés de 14 à 17 ans et placés pour des problèmes de comportement ont participé à cette étude. Ces jeunes, placés depuis en moyenne 5,6 ans, ont été identifiés comme étant résilients (à partir d'une grille soumise aux intervenants et d'une discussion clinique). Deux entrevues ont été menées avec

1. Cette recherche a été rendue possible grâce à une subvention de recherche du Fonds Richelieu de recherche sur l'enfance et du soutien financier du Centre jeunesse de Québec-Institut universitaire sur les jeunes en difficulté.

2. Centre de recherche sur l'adaptation des jeunes et des familles à risque, Université Laval, Québec.

Service social, volume 51, numéro 1, 2004-2005 
chaque jeune. Les résultats mettent en évidence les facteurs de protection environnementaux (présence de liens significatifs avec des adultes, notamment des intervenants, services de qualité et sources de valorisation) et individuels (confiance en soi, perception de contrôle, stratégies d'adaptation) qui ont favorisé la résilience de ces jeunes.

\section{Mots clés : résilience, adolescence, hébergement en milieu substitut.}

The main goal of this qualitative research is to shed light on the protection and vulnerability factors in teenagers who are living in foster care and considered to be resilient. Twelve young people (6 boys and 6 girls), who were from 14 to 17 years old and who had been placed in homes because of behavioural problems, participated in this study. These young people, who had been in placements for an average of 5.6 years, were identified as being resilient based on a grid filled out by practitioners and on a clinical discussion. Two interviews were held with each teenager. The results point to environmental protection factors (significant relationships with adults, particularly practitioners, quality services, and sources of approval) as well as individual factors (will to succeed in life, perception of control, adaptation strategies) that fostered these young people's resilience.

Keywords: resilience, adolescent, foster care.

\section{INTRODUCTION}

Pourquoi certains jeunes aux prises avec de grandes difficultés personnelles et familiales, et malgré un contexte de vie difficile, s'en tirent-ils mieux que d'autres qui pourtant sont exposés aux mêmes conditions de vie (pauvreté, négligence, toxicomanie, agressions physiques ou sexuelles)? Ces jeunes qui s'en sortent malgré les coups du sort sont appelés depuis quelques années des jeunes résilients. L'objectif général de cette recherche est de mettre en lumière tant les facteurs de protection que les facteurs de vulnérabilité présents chez des adolescents placés considérés comme étant résilients.

II apparaît plus que pertinent de se pencher sur la problématique des adolescents en contexte de placement, étant donné la situation de grande vulnérabilité dans laquelle ces jeunes se trouvent, notamment à l'issue du placement: problèmes de sousscolarisation, occasions d'emploi restreintes, manque de soutien familial, problèmes de santé mentale, risque d'itinérance, toxicomanie. Ces problèmes ont été constatés aux États-Unis (Courtney, Piliavin, Grogan-Kaylor et Nesmith, 2001), au Canada (Pauzé, Toupin, Déry et Mercier, 2000) et ailleurs dans le monde (Weiner et Kupermintz, 2001). Les jeunes placés sont, en effet, parmi les groupes sociaux les plus vulnérables en raison de l'ampleur et de la complexité des problèmes auxquels ils sont confrontés dans leur milieu familial d'origine, des conditions sociales et économiques adverses dans lesquelles ils se trouvent et de la présence de difficultés souvent très importantes chez leurs parents (Brady et Caraway, 2002; Pauzé et al., 2000). Ajoutons aussi que le placement, tout en étant une mesure de protection puisqu'on retire le jeune d'un milieu 
constituant une menace pour sa sécurité ou son développement, entraîne, à certains égards, des risques additionnels. C'est notamment le cas en ce qui concerne les ruptures de lien avec des personnes significatives et les multiples déplacements à l'intérieur même des ressources (Cloutier, 2000; Rutter, 2000).

On observe, par ailleurs, une grande hétérogénéité dans l'adaptation des jeunes hébergés en milieu substitut. En effet, tous ne se maintiendront pas dans une trajectoire problématique (Rutter, 2000; Weiner et Kupermintz, 2001). La présente recherche se situe dans ce courant d'études et vise à approfondir notre compréhension des facteurs qui contribuent à ce que certains adolescents hébergés en milieu substitut s'en sortent mieux que d'autres.

\section{CADRE THÉORIQUE}

\section{FACTEURS DE RISQUE, FACTEURS DE PROTECTION ET RÉSILIENCE}

Généralement, la résilience est définie comme la présence ou le maintien de compétences malgré un contexte de risque (Masten et Coatsworth, 1998); la compétence est alors présentée comme une adaptation réussie à l'environnement. Cela étant dit, il est essentiel de se demander ce que sont une compétence et une adaptation réussie. En effet, les comportements jugés compétents et adaptés dans un environnement donné peuvent être considérés comme étant tout à fait inadaptés dans un autre. Par exemple, un jeune peut développer des comportements antisociaux dans un contexte où les actes délinquants confèrent un statut social élevé. Afin de contrer cette difficulté à définir l'adaptation, les auteurs font généralement référence à un certain nombre de compétences nécessaires à la poursuite du développement pour porter un regard sur la résilience (ex. : capacité d'attachement, autocontrôle, adaptation scolaire, établissement de liens d'amitié). Ainsi, dans l'exemple donné plus haut, les comportements antisociaux du jeune, bien qu'ils puissent être une stratégie de survie dans son environnement, ne seraient pas considérés comme reflétant la résilience, sur le plan comportemental tout au moins. Les qualités de débrouillardise ou d'intelligence qui sont peut-être associées à cette stratégie pourraient être, par ailleurs, des points d'appui sur lesquels se développera la résilience de ce jeune.

Ainsi, la nature multidimensionnelle du concept de résilience est désormais reconnue. En effet, l'évaluation de diverses sphères de compétence a permis de constater qu'une proportion relativement faible des enfants est résiliente dans toutes les sphères; pour la majorité, la résilience s'observe dans quelques domaines spécifiques (Luthar, 1997). Ainsi, un jeune peut très bien réussir sur le plan scolaire, mais être moins compétent sur le plan social.

Les termes risque, vulnérabilité et protection étant associés à la résilience, il s'avère essentiel de les définir. Tout d'abord, le risque prédispose les individus ou les groupes à l'apparition de conséquences négatives. Le risque résulte d'un traumatisme, d'un événement de vie majeur ou de stresseurs chroniques. La vulnérabilité consiste pour 
sa part en une augmentation de la probabilité d'apparition de conséquences négatives en présence du risque. Donc, la notion de vulnérabilité n'a de sens qu'en présence du risque. Finalement, à l'inverse, la protection diminue la probabilité d'apparition des conséquences négatives, toujours en présence du risque.

D'une manière générale, les facteurs de protection qui favorisent la résilience sont assez bien connus. Ils sont de trois ordres : individuel, familial et environnemental. Les facteurs individuels sont notamment l'intelligence, les habiletés sociales, l'estime de soi, la perception de contrôle, la résolution de problème, l'empathie et l'espérance. Les facteurs familiaux sont, entre autres, les liens affectifs soutenants dans la famille, les attentes positives envers l'enfant, un style parental démocratique, la santé mentale des parents et les liens avec le réseau familial étendu. Sur le plan environnemental, on retrouve notamment les liens avec des adultes «pro sociaux » et la fréquentation d'institutions qui soutiennent les compétences des jeunes. Si ces facteurs sont connus de manière générale, il reste qu'ils ne s'appliquent pas nécessairement à tous les contextes de risque ni à tous les enfants. Une compréhension du rôle de ces facteurs nécessite donc une évaluation complexe des contextes de vie des jeunes.

\section{DES JEUNES RÉSILIENTS EN CONTEXTE DE PLACEMENT}

À notre connaissance, seules quelques études ont porté spécifiquement sur la résilience des enfants et des adolescents en contexte de protection de la jeunesse. À ce chapitre, mentionnons les travaux réalisés par Rutter et ses collaborateurs (Rutter et al., 1995; Rutter et Quinton, 1984). À la suite de leurs rencontres avec des adultes ayant vécu en institution durant leur enfance, ces auteurs affirment que le fait de pouvoir vivre des expériences positives durant l'enfance augmente la perception de contrôle du jeune face à sa vie. Cette perception favorise, à son tour, une meilleure planification de vie, particulièrement dans le domaine professionnel. Jackson et Martin (1998) se sont eux aussi intéressés aux adultes ayant vécu en institution durant leur enfance. Ils concluent que le succès scolaire est un facteur déterminant du style de vie et de l'insertion sociale réussie de ce groupe de jeunes.

Deux autres recherches apportent aussi des informations complémentaires aux précédentes, puisqu'elles ont porté sur des jeunes suivis par les services de protection au moment même de l'étude. Ainsi, Lösel et Bliesener (1994) ont réalisé une étude quantitative offrant un portrait des caractéristiques d'adolescents résilients hébergés en centre d'accueil. Selon cette étude, les jeunes résilients sont plus intelligents, plus flexibles, ont une estime de soi positive, se sentent plus efficaces, plus actifs et se perçoivent comme ayant un contrôle sur leur vie. Ils entretiennent aussi une perception plus réaliste du futur. La présence, auprès de ces jeunes, de personnes significatives extérieures à la famille a également été notée. On peut déplorer cependant le fait que ces résultats offrent un portrait statique et surtout individuel du jeune résilient, laissant dans l'ombre les dimensions environnementales. Pour sa part, Henry (1999) a étudié, à l'aide d'une méthodologie qualitative, la résilience d'adolescents suivis par les services de protection. Les sept jeunes composant l'échantillon ont été interviewés à trois reprises afin d'approfondir les stratégies déployées vis-à-vis des agressions 
vécues durant leur enfance. Les résultats font ressortir cinq grands thèmes, soit la capacité de ces jeunes à : 1) ne pas se blâmer; 2) normaliser la situation; 3) prendre une distance par rapport à la situation; 4) se voir comme des personnes ayant une valeur; 5) croire en leur futur.

Bref, nous en savons encore peu sur la résilience des jeunes en contexte de placement. La présente recherche, réalisée dans une approche qualitative, contribue à l'avancement des connaissances dans ce champ en approfondissant le point de vue des jeunes eux-mêmes.

\section{MÉTHODOLOGIE}

\section{POPULATION À L'ÉTUDE ET CRITÈRES D'IDENTIFICATION DES JEUNES RÉSILIENTS}

La population à l'étude était composée de jeunes entre 14 et 17 ans pris en charge par le Centre jeunesse de Québec en vertu de la Loi sur la protection de la jeunesse. Ces jeunes devaient être placés depuis au moins trois mois à la suite d'une décision de placement, cela afin d'éviter les mesures provisoires ou de dépannage, et résider en centre de réadaptation, en foyer de groupe ou en famille d'accueil. Afin de trouver les adolescents et adolescentes susceptibles de participer à cette étude, nous avons sollicité la collaboration des intervenants. À cet effet, une grille a été bâtie afin de préciser, de manière opérationnelle, les critères de définition de la résilience. La grille présente cinq domaines de compétence précisés par des indicateurs (voir tableau 1). Ces domaines de compétence, ainsi qu'une première liste d'indicateurs possibles, ont été tirés des écrits du domaine (Luthar, 1997; Masten et Coastworth, 1998). Par la suite, ces indicateurs ont été raffinés en collaboration avec deux des auteurs du présent article qui sont des intervenants d'expérience dans les centres de protection de la jeunesse. À cette étape nous avons pu opérationnaliser le concept de résilience en tenant compte du contexte de placement, nous accordant ainsi avec les auteurs qui soulignent la nécessité de définir la résilience à la lumière du contexte particulier dans lequel les personnes vivent (Fraser, Richman et Galinsky, 1999). Autrement dit, les jeunes résilients sélectionnés pour cette étude n'avaient pas nécessairement à atteindre un niveau de compétence égal ou supérieur à celui des adolescents de la population générale, mais bien à se situer à un niveau de compétence supérieur à celui de leur propre groupe de référence, soit les jeunes hébergés en milieu substitut. 
Tableau 1

Domaines et indicateurs de la résilience chez les jeunes hébergés en milieu substitut

\begin{tabular}{|c|c|}
\hline Domaines & Indicateurs \\
\hline $\begin{array}{l}\text { Formation scolaire ou } \\
\text { préparation à l'emploi }\end{array}$ & $\begin{array}{l}\text { - Va à l'école ou participe à un programme d'employabilité; } \\
\text { - Fait des apprentissages; } \\
\text { - Est motivé à réussir et fait des efforts; } \\
\text { - Se comporte bien à l'école et dans le milieu de stage. }\end{array}$ \\
\hline Relations avec les pairs & $\begin{array}{l}\text { - Entretient des relations avec un groupe d'amis socialement } \\
\text { acceptables; } \\
\text { - Est capable de vivre une relation amoureuse saine; } \\
\text { - A au moins un ami intime de même sexe. }\end{array}$ \\
\hline Relations avec les adultes & $\begin{array}{l}\text { - Est capable d'établir un lien significatif avec un adulte } \\
\text { positif; } \\
\text { - A des contacts positifs avec des personnes à l'intérieur ou } \\
\text { à l'extérieur du milieu de placement. }\end{array}$ \\
\hline Caractéristiques personnelles & $\begin{array}{l}\text { - Se fixe des buts positif;; } \\
\text { - Se reconnaît des forces; } \\
\text { - S'accorde de la valeur (prend soin de lui ou d'elle); } \\
\text { - Fait preuve d'autonomie; } \\
\text { - A le sens de l'humour; } \\
\text { - Est capable d'aller chercher et d'accepter l'aide dont il ou } \\
\text { elle a besoin. }\end{array}$ \\
\hline Comportements & $\begin{array}{l}\text { - Se valorise à travers des activités sportives, culturelles ou } \\
\text { autres à l'intérieur ou à l'extérieur du milieu de placement; } \\
\text { - A des comportements socialement acceptables et se con- } \\
\text { forme aux règles du milieu où il ou elle vit actuellement. }\end{array}$ \\
\hline
\end{tabular}

Pour chacun des items, l'intervenant évaluait le jeune à partir d'une échelle Likert. Une question ouverte terminait l'instrument, permettant ainsi à l'intervenant d'expliquer son évaluation. Pour le qualifier de résilient, nous avons établi à trois sur cinq le nombre de domaines de compétence sur lesquels un jeune devait se distinguer. Par la suite, une discussion avec l'intervenant permettait de statuer sur la situation du jeune.

\section{DESCRIPTION DE L'ÉCHANTILLON}

La méthode d'échantillonnage est de type intentionnel. Cette méthode s'applique à l'étude de cas retenus en raison de leur exemplarité, plutôt qu'au caractère représentatif d'une population au sens statistique. Dans une perspective qualitative, c'est l'accès à un matériel riche et approfondi qui favorise la mise en lumière de processus fondamentaux, plutôt que la quantité de cas examinés (Laperrière, 1997). Afin de constituer l'échantillon, 66 grilles ont été remplies par les intervenants. De ce nombre, seules 18 correspondaient aux critères de résilience retenus, soit $27 \%$ des grilles remplies. Parmi celles-ci, deux correspondaient à des jeunes qui étaient de retour à leur domicile familial et une à un jeune qui était en fugue au moment du recrutement, ce qui les excluait de l'étude. Enfin, trois jeunes ont refusé de participer. 
L'échantillon final est donc composé de douze jeunes placés, soit six garçons et six filles, âgés de 14 à 17 ans et suivis en vertu de la Loi sur la protection de la jeunesse (LPJ). L'âge moyen des jeunes est de 15,9 ans. Ces jeunes ont été placés durant au moins trois mois. Au moment des entrevues, la majorité des jeunes vivaient en centre de réadaptation $(n=10)$, un jeune était placé en foyer de groupe et un autre vivait en famille d'accueil spécifique. Dans la majorité des cas $(n=11)$, le motif principal du dernier placement se rapportait à des troubles ou problèmes de comportement sérieux. Le tableau 2 présente les caractéristiques des participants.

Tableau 2

Histoires socio-familiale et de placement $(N=12)$

\begin{tabular}{|c|c|c|c|}
\hline $\begin{array}{l}\text { Caractéristiques personnelles } \\
\text { et familiales des jeunes }\end{array}$ & $\begin{array}{l}\text { Filles } \\
(n=6)\end{array}$ & $\begin{array}{c}\text { Garçons } \\
(n=6)\end{array}$ & $\begin{array}{c}\text { Total } \\
(N=12)\end{array}$ \\
\hline Âge moyen des jeunes & 16,2 ans & 15,5 ans & 15,9 ans \\
\hline 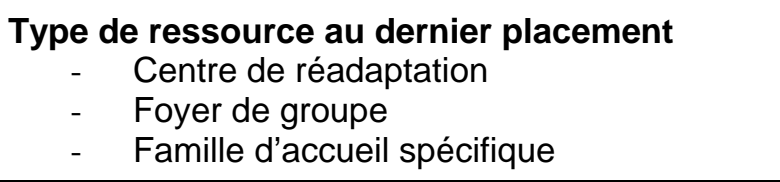 & 6 & $\begin{array}{l}4 \\
1 \\
1\end{array}$ & $\begin{array}{r}10 \\
1 \\
1\end{array}$ \\
\hline $\begin{array}{l}\text { Durée totale de la trajectoire de placement } \\
-\quad 6 \text { ans et moins } \\
-\quad 7 \text { ans et plus }\end{array}$ & $\begin{array}{l}3 \\
3\end{array}$ & $\begin{array}{l}4 \\
2\end{array}$ & $\begin{array}{l}7 \\
5\end{array}$ \\
\hline $\begin{array}{c}\text { Motif principal du dernier placement } \\
-\quad \text { Troubles de comportement sérieux } \\
\text { - } \quad \text { Négligence parentale }\end{array}$ & $\begin{array}{l}5 \\
1\end{array}$ & $\begin{array}{l}5 \\
1\end{array}$ & $\begin{array}{r}10 \\
2\end{array}$ \\
\hline 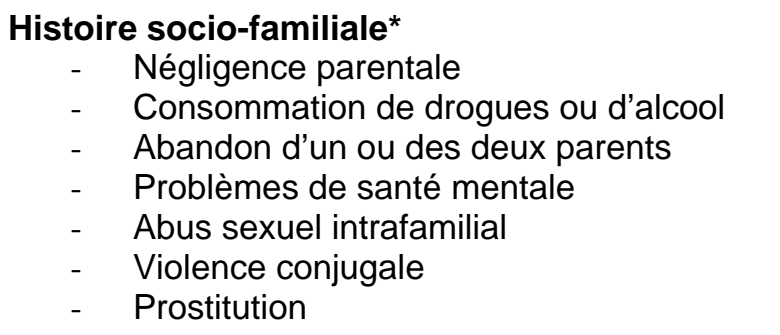 & $\begin{array}{l}6 \\
3 \\
3 \\
3 \\
4 \\
4 \\
-\end{array}$ & $\begin{array}{l}5 \\
4 \\
3 \\
3 \\
1 \\
- \\
1\end{array}$ & $\begin{array}{r}11 \\
7 \\
6 \\
6 \\
5 \\
4 \\
1\end{array}$ \\
\hline $\begin{array}{cl}\text { Motifs du dernier signalement retenu } \\
\text { - } & \text { Victime de négligence parentale } \\
\text { - } & \text { Troubles de comportement sérieux } \\
\text { - } & \text { Victime de négligence et d'abus sexuel } \\
\text { - } & \text { Victime de négligence et d'abus physique } \\
\text { - } & \text { Négligence, troubles de comportement et } \\
& \text { abus sexuel sur d'autres jeunes } \\
\text { - } & \text { Abandon par les deux parents }\end{array}$ & $\begin{array}{l}2 \\
3 \\
1 \\
- \\
-\end{array}$ & $\begin{array}{l}1 \\
- \\
1 \\
1 \\
2 \\
1\end{array}$ & $\begin{array}{l}3 \\
3 \\
2 \\
1 \\
2 \\
1\end{array}$ \\
\hline
\end{tabular}

* Situation présentée par un ou par les deux parents. 


\section{INSTRUMENT}

Aux fins de la présente étude, deux entrevues ont été menées avec chaque jeune. La première entrevue, de type ouvert, a permis d'aborder le jeune à partir de questions générales qui apportent des renseignements sur l'ensemble de sa vie. Par exemple, les premières questions étaient: 1) « Un journaliste veut écrire un article sur toi. II aimerait que tu lui racontes ta vie. »2) «Dans ta vie, quelle est la chose la plus importante qui te soit arrivée? » La deuxième entrevue, de type semi-dirigé, était axée sur des thèmes plus précis afin de centrer les propos du jeune sur des éléments ayant pu jouer un rôle dans l'actualisation de la résilience. Des questions portaient notamment sur les forces du jeune, son réseau de soutien familial et extrafamilial, ses stratégies d'adaptation, ses buts. La planification de la deuxième entrevue a nécessité une première analyse des entrevues ouvertes afin de cibler différents aspects importants dans la vie de chaque jeune.

\section{ANALYSE DES DONNÉES}

Toutes les entrevues ont été enregistrées et retranscrites. Une analyse de contenu thématique a été privilégiée. Cette analyse consiste à repérer, dans les propos des répondants, des thèmes généraux qui apparaissent sous divers contenus plus concrets. Ces thèmes sont ensuite organisés en catégories d'analyse. Un modèle mixte a été utilisé lors de l'élaboration du système de catégorisation du matériel. En effet, des catégories ont émergé du discours des jeunes, alors que d'autres, nommément celles relatives au processus menant à la résilience, ont été préalablement identifiées à partir du modèle proposé par Rutter (1990). Le système de catégorisation s'est par la suite raffiné de manière itérative à partir de l'analyse des entrevues menées auprès de six participants. Certaines catégories préliminaires ont alors été retirées ou regroupées et d'autres se sont ajoutées afin de mieux rendre compte des propos des jeunes. Les règles qui ont présidé au choix des catégories retenues sont la pertinence au regard des objectifs de l'étude, l'exhaustivité, la représentativité et l'homogénéité (Mayer, Ouellet, Saint-Jacques et Turcotte, 2000). Notons que l'apport des intervenants engagés dans ce projet a été particulièrement précieux afin d'interpréter le sens des propos des jeunes.

Les entrevues ont ensuite été codifiées par une des auteurs à l'aide du logiciel NUD*IST (version 4.0). Les entrevues ont été analysées tout d'abord verticalement afin de repérer les thèmes dans chacune d'elles, puis elles ont fait l'objet d'une analyse horizontale qui permet des comparaisons entre les cas. L'accord inter-juges, calculé à partir de $17 \%$ du matériel codifiable, est de $79 \%$. Ce niveau d'accord est supérieur à ce qui est jugé acceptable en recherche qualitative (Boyatzis, 1998). 


\section{RÉSULTATS $^{3}$}

\section{LES JEUNES RACONTENT UNE HISTOIRE MARQUÉE PAR DE MULTIPLES RISQUES}

\section{Les contextes familiaux}

La négligence parentale, les abus physiques et sexuels, les problèmes de santé mentale, la consommation de drogue et d'alcool, l'incarcération d'un parent, les conflits conjugaux et la violence familiale sont couramment mentionnés par les jeunes rencontrés. L'extrait suivant illustre le passé difficile d'une jeune, mais aussi celui de ses parents.

Quand je suis née, tout allait bien. Puis, après ça, mes parents ont commencé à se chicaner, mon père n'était jamais là. Ma mère, elle était tout le temps toute seule. [...] Elle trouvait ça difficile, donc elle est partie à un moment donné. Elle est allée me porter chez sa sœur. [...] Elle est venue me porter là mais mon père est venu nous chercher. Mais là, il a mis le feu dans le logement, et lui il nous a laissés là. (Florence)

Plus spécifiquement, lorsqu'ils racontent l'histoire de leur famille, le premier événement marquant que les jeunes mentionnent se rapporte soit à la séparation de leurs parents, soit au fait qu'ils n'ont jamais pu vivre avec leurs vrais parents, ayant connu très tôt un premier placement dans une famille d'accueil. En outre, la majorité des jeunes n'ont jamais connu leur père, alors que les autres n'ont que des contacts sporadiques ou inexistants avec ce dernier. Ce premier événement marquant s'accompagne, pour certains, d'un sentiment d'abandon, alors que chez d'autres on note un sentiment de frustration et de colère à l'égard d'un parent qu'ils ont, somme toute, peu ou même jamais connu.

Bien, à partir de mettons 7 ans, mon père a commencé à avoir une blonde, puis là, de plus en plus, il allait tout le temps chez elle. À un moment donné, ils ont déménagé les deux, puis lui, il nous a acheté un appartement à moi puis à ma soeur. Il était tout le temps rendu chez sa blonde. Puis nous autres, il s'en foutait pas mal. C'est comme genre moi qui a fait vivre ma petite sœur. (Manuel)

La plupart des jeunes mentionnent aussi une absence de règles à la maison, un manque de suivi et d'encadrement dans les horaires ou les travaux scolaires et, dans certains cas, un rejet affectif grave et continu.

Ça n'allait pas bien. Elle ne s'occupait pas de moi. J'habitais là, mais j'étais comme un pensionnaire, je faisais ce que je voulais et je rentrais à n'importe quelle heure. (Françoise)

3. Afin de garder l'anonymat des jeunes, tous les prénoms ont été changés. De plus, tous les indices permettant de reconnaître les situations ont été enlevés. 
Finalement, des problèmes relationnels importants entre le jeune et son parent (agressivité verbale et physique, opposition, fugues, vols) sont également mentionnés par tous les jeunes qui vivaient dans leur famille naturelle avant leur dernier placement.

Mes parents, je les envoyais chier. C'était vraiment l'enfer. La violence. Je pétais tout dans la maison. Eux autres, ils m'ont mis en famille d'accueil de dépannage. J'étais supposé être là juste un mois, puis en fin de compte je suis resté là huit mois. Mes parents ne voulaient pas me reprendre encore, parce qu'ils disaient que je n'avais pas changé. (Marcel)

\section{L'histoire de placement}

Les jeunes relatent des histoires de placement qui leur paraissent parfois très longues. Par exemple, Marco, 17 ans, placé depuis l'âge de 10 ans, raconte : " J'ai passé ma vie en centre d'accueil. Je commence à être tanné, j'ai hâte de sortir. Je suis inquiet. »

Ces histoires sont souvent marquées par de multiples déplacements d'une ressource à l'autre, parfois entrecoupés de tentatives infructueuses de réinsertion dans le milieu familial. L'histoire de placement constitue, dès lors, une source de frustration importante, voire de ressentiment pour le jeune. "Je suis tannée de faire plusieurs places : familles d'accueil, ma grand-mère, foyer de groupe... Je suis tannée. J'espère que ça va être le dernier parce que je suis tannée. Je suis vraiment tannée. » (Florence)

\section{Les difficultés personnelles}

Au cours des entrevues, les jeunes ont témoigné de leurs difficultés personnelles. À ce chapitre, la majorité des jeunes mentionnent des problèmes liés à une consommation abusive de drogue ou d'alcool, problèmes qui sont d'ailleurs souvent à l'origine du placement actuel.

Bien ça allait mal. Je me battais avec du monde souvent. Donc je me faisais mettre à la porte des écoles. J'ai commencé à consommer un peu plus. Quand je suis revenu ici par après, encore à cause de l'école et chez nous, j'ai recommencé à consommer. [...] Je n'avais plus de fun. Je consommais tout le temps, tout le temps. (Maxime)

Des relations avec des pairs antisociaux ou avec de jeunes adultes déviants sont aussi fréquentes. Ces relations sont associées à des problèmes de consommation de drogue et d'alcool et à la délinquance juvénile.

On a recommencé à se voir, on était tout le temps ensemble. On allait à la même école. J'ai commencé à fumer la cigarette, j'ai commencé à boire, j'ai commencé à fumer du pot. [...] Ça a commencé. Je n'allais plus à l'école, puis là les vols ont commencé. (Marcel)

Finalement, tous les jeunes ont rapporté, à un moment ou à un autre dans leur histoire, avoir vécu des difficultés scolaires. Des problèmes tels que l'absentéisme scolaire, les difficultés relationnelles avec les pairs, les difficultés d'apprentissage et la démotivation sont souvent mentionnées. 


\section{LES FACTEURS DE PROTECTION}

\section{Les facteurs de protection environnementaux}

\section{Des liens significatifs positifs avec des adultes}

L'analyse des entrevues permet de constater à quel point la présence d'un lien significatif positif avec un ou des adultes joue un rôle important dans la résilience des jeunes hébergés en milieu substitut. Si ce lien n'est pas établi directement auprès d'un membre de la famille immédiate (mère, beau-père ou fratrie), on trouvera une tante, un cousin, un entraîneur, un professeur ou un autre adulte avec qui le jeune a établi un lien de confiance. En fait, tous les jeunes rencontrés ont mentionné au moins un lien significatif avec les membres de leur entourage. Ces adultes jouent ainsi un rôle clé pour soutenir et orienter les jeunes dans la reprise de leur développement. Plus spécifiquement, la mère demeure une source de soutien incontournable dans la vie de la plupart des jeunes qui ont participé à cette étude, et ce, malgré les contextes familiaux difficiles. Les extraits qui suivent illustrent l'importance de l'implication de ce parent dans la résilience des adolescents.

Bien que ma mère, elle soit encore avec moi, qu'elle ne me lâche pas. Parce qu'elle aurait pu dire : «moi, je la place en famille d'accueil puis je ne m'occupe plus d'elle ». Mais, elle n'est pas de même. (Françoise)

Ma mère, elle est venue assister à tous les spectacles que j'ai faits, à toutes les pièces de théâtre.[...] Ma mère, elle a toujours été présente pour m'encourager. (Mathieu)

La présence d'un beau-parent, d'un membre de la fratrie ou de la parenté avec qui le jeune a une relation positive peut également contribuer à maintenir les compétences du jeune.

Mon beau-père, je suis bien contente de l'avoir. C'est la première fois, dans toutes mes années de vie, que j'ai un père comme ça, qui s'occupe de ma petite sœur et qui se préoccupe de moi. Ça me rend vraiment heureuse. [...] Je peux lui dire des choses, lui confier des choses qu'il ne dira pas à personne. C'est vraiment fiable. Je peux avoir confiance. (Francine)

Oui, ma mère et ma tante. Ça, c'est comique parce que ma tante, elle parle vraiment beaucoup. Elle m'aide quasiment à régler tout. Elle a vécu les mêmes affaires. (Fabienne)

Pour d'autres, on remarque la présence de liens positifs qui se sont créés à l'extérieur de la famille immédiate ou élargie. Par exemple, Manuel, placé en raison d'abandon, a trouvé dans son entraîneur une personne qui est devenue « tuteur de remplacement ». « J'en parle plus à mon professeur de karaté. J'y parle de tout à lui. Puis il sait déjà tout. [...] Quand je parle à mon entraîneur, c'est comme si c'était à mon père. » 
Par ailleurs, il n'est pas toujours nécessaire pour un adulte d'être un tuteur de remplacement pour exercer une influence significative dans la trajectoire d'un jeune. Par exemple, dans ce cas, le professeur a joué un rôle particulier dans la démarche du jeune. L'attention qu'il lui a portée a été significative et ses propos ont eu un sens particulier pour ce jeune, notamment en l'amenant à réfléchir aux conséquences de ses actes.

Moi, je trouve que c'est quand j'allais à l'école que c'est là que j'ai commencé à réaliser des affaires. On a des cours de FPS, puis la madame, souvent, elle fermait la porte, puis elle me parlait parce que j'avais plein de problèmes, plus que les autres. Puis là elle m'a parlé d'empathie, je pense que c'est à cause de ça. (Martin)

\section{Des intervenants et des services}

Les jeunes se sentent soutenus, non seulement par leur famille et leurs amis, mais également par les intervenants qu'ils ont rencontrés. Ils réalisent que les gens autour d'eux souhaitent qu'ils s'en sortent.

C'est plus alentour de moi, des personnes qui voulaient m'aider comme les éducateurs, ma mère, mes amis, puis tout ça, qui ont comme formé un cercle, puis moi j'étais dans le milieu, puis ils me disaient tous : « Sors-toi de là. » C'est plus comme ça. Ces personnes-là, elles ont tassé les aspects négatifs, les personnes négatives pour moi. C'était juste du positif. (Francine)

Les éducateurs, ce que je retiens de mon expérience, c'est que c'est des gens tellement dévoués, puis que c'est vraiment du bon monde. Je pense qu'ils m'ont vraiment aidée. Je ne pourrai jamais assez les remercier. [...] Les éducateurs, il faudrait leur rendre hommage. (Fanny)

Tous ne sont cependant pas aussi positifs. Certains jeunes sont en effet très critiques face aux intervenants, sans pour autant les mettre tous dans le même panier. De fait, même dans ces situations, on trouvera généralement au moins un intervenant à qui le jeune fait confiance et à qui il reconnaît une valeur. Les jeunes ont alors l'impression que ces intervenants s'intéressent réellement à eux et qu'ils croient en leur capacité de s'en sortir.

Des fois, il y en a qui me gossaient en hostie... C'est parce que ces gens-là, ils se prennent pour nos mères, ils veulent nous protéger, mais dans le fond ils nous font juste chier et péter notre coche. Mais il y en a une avec qui j'ai eu des rencontres, elle est crissement smatt. Elle ne me fait pas chier et elle veut vraiment que je retourne chez nous. (Marcel)

Au-delà des liens significatifs établis avec certains intervenants, les jeunes reconnaissent également le rôle proprement dit des services offerts dans l'établissement. Ces services favorisent le cheminement des jeunes vers une prise de conscience ou, dans certains cas, consolident leur désir de changement. 
Oui, c'était le transfert des acquis [nom du programme]. C'était le lundi soir. À un moment donné, à force d'assister à ça, veut ou veut pas, tu te mets à écouter ce qu'ils ont à dire. J'ai acquis ça pas mal au moins. (Maxime)

Puis j'ai fait une thérapie fermée. Je pense que ça m'a donné un coup de pouce. Peut-être pas tant que ça, mais tu sais, juste au moins savoir que je ne suis pas toute seule sur la terre pognée de même, c'est déjà pas si mal! (Fanny)

\section{Des sources de valorisation}

Dans cette étude, presque tous les jeunes rencontrés ont pu décrire des expériences qui ont été valorisantes pour eux. Ces expériences sont d'ordre sportif, artistique, professionnel ou autre.

Je me suis mis à faire des compétitions pas mal partout [...]. J'ai été champion [...].C'est quand j'ai été champion. Ça m'a apporté le bonheur. (Manuel)

Ma pièce de théâtre qui vient juste de partir. Disons que j'avais mis beaucoup de cœur. Je suis le seul gars en dehors de la scène qui est du genre à rester après les cours. [...] Je m'implique beaucoup. (Mathieu)

J'ai participé à ça [carrière-étude] cet été. [...] J'ai eu mon évaluation, puis c'était tout excellent. Même la ponctualité, c'était triple excellent. J'avais tout le temps une heure d'avance, puis tout ça. C'était le fun. Je me donne à $100 \%$. (Francine)

Ces expériences significatives ont contribué au maintien ou au développement de l'estime de soi chez les jeunes. Par exemple, Marco mentionne: "Quand je réussis quelque chose que je veux vraiment réussir. Je veux le réussir, puis quand je le réussis, je suis content. »

\section{Les facteurs de protection internes}

\section{La confiance en soi}

La confiance en soi est fréquemment mentionnée par les jeunes de l'étude. On peut le constater dans les deux extraits suivants :

Je pense la confiance en soi. Je pense que c'est ça qui m'a aidé. Si je n'avais pas vraiment eu confiance, je n'aurais pas été capable de le faire. (Martin)

Je compte sur moi. La confiance pour continuer à réussir, puis être capable de faire ce qu'il me reste à faire. (Florence)

En outre, les répondants sont conscients de leurs qualités personnelles. Ils les mentionnent avec aisance au cours des entrevues. Ils se considèrent généralement comme des personnes gentilles et généreuses et constatent que ces qualités sont très importantes, puisqu'elles leur servent à s'intégrer au groupe. 
Beau, fin, gentil... Non, mais sérieux, je suis fin, je suis généreux. Vous le demanderez à ma mère la prochaine fois que vous lui parlerez. Je suis très serviable. (Mathieu)

Je suis une personne très généreuse. Ma mère me le dit tout le temps. Moi, en tout cas, je trouve que je suis généreuse puis intelligente. (Fanny)

Certains savent, par expérience, qu'ils sont capables de se débrouiller peu importe la situation. Par exemple, Marcel affirme : «Bien je suis débrouillard certain! [Même en fugue] on bouffait au resto trois fois par jour, on allait aux arcades, on se roulait des joints, on était logés nourris! »

\section{Les stratégies d'adaptation}

L'utilisation de certaines stratégies d'adaptation en présence d'événements stressants peut également constituer un facteur de protection. Parmi les stratégies mentionnées par les jeunes, on trouve à la fois des stratégies centrées sur le problème et des stratégies centrées sur les émotions. Plus particulièrement, les jeunes ont souvent fait référence à des stratégies de résolution de problème. Par exemple, cette jeune qui nous dit: " Je prends le temps de réfléchir, je respire puis je prends tous les moyens. Des fois j'écris sur une feuille, les pour et les contre. » (Françoise).

Se changer les idées et relaxer sont aussi des stratégies très fréquentes chez les jeunes participants de cette étude. De l'avis même des jeunes, ces stratégies peuvent leur éviter bien des ennuis. "J'ai ma guitare. Quand je suis frustré, je pars en débile, puis je gueule. J'écris les paroles, c'est fucké. [...] Ça m'aide à gérer mes émotions... Genre si je suis frustré, je joue. Je suis là, je gueule comme un malade. Genre si je suis triste, je joue des petites affaires. » (Marcel)

Voir le bon côté d'une situation et accepter leur vie et les expériences qu'ils ont vécues sont aussi des stratégies rapportées par quelques jeunes. L'extrait suivant illustre ces stratégies.

Toutes les expériences que j'ai vécues dans ma vie, qu'elles soient bonnes ou mauvaises, ça m'a retiré une leçon. C'est avec ça aussi que j'avance plus dans ma vie, puis que je suis capable d'y voir plus clair, puis que ça c'est pas bon pour moi, puis ça c'est bon, qu'est-ce que je veux dans la vie. [...] J'ai pu vivre certaines choses qu'il y en a qui n'ont pas vécues. (Francine)

Quelques jeunes, dont Martin, mentionnent également la demande d'aide comme étant une stratégie employée lorsqu'ils vivent des difficultés : " Je vais aller consulter. Faut que je me calme. Si je ne suis pas capable, sois je la pète ou j'en parle à quelqu'un. Je me suis rendu compte que, quand tu en parles, ça décompresse. » 


\section{La perception de contrôle sur sa vie}

Une impression de contrôle réaliste a régulièrement été identifiée comme un facteur de protection dans les écrits portant sur les jeunes résilients. Les propos rapportés par les jeunes de la présente étude confirment l'importance de ce facteur.

Je le sais que tout ce que je fais, c'est la conséquence de mes actes qui m'arrive. [...] Puis oui, aussi j'ai le contrôle de ma vie parce que je subis les conséquences de mes actes. Je me dis, c'est moi qui contrôle mes actes. Je ne contrôle pas les conséquences, mais je contrôle les actes, puis les conséquences vont avec les actes. (Fanny)

C'est sûr que j'ai plus de contrôle parce que c'est moi qui dirige ça. Si mettons je fais quelque chose, ça va être de ma faute. Mais ça peut être positif... Comment je pourrais dire? Parce que dans le fond, je me suis aidée, puis je commence, je vais sortir bientôt du centre d'accueil. Je sais que c'est moi qui fais le choix de sortir parce que c'est moi qui a fait le choix d'aller bien. (Francine)

\section{LES ZONES DE VULNÉRABILITÉ}

\section{Les caractéristiques personnelles}

Les jeunes mentionnent régulièrement l'impatience, l'agressivité, l'impulsivité et la naïveté comme faisant partie de leur personnalité. Ces caractéristiques, une fois combinées avec les risques de leur environnement, sont susceptibles d'entraver le processus de changement amorcé. À titre illustratif, citons Marcel qui affirme: "Bien des fois, je pète ma coche pour rien. Ça dépend des fois, je suis trop impulsif vite. »

Pour d'autres jeunes, notamment ceux qui ont vécu l'abandon d'un parent, on note la difficulté de faire confiance aux adultes. Dans bien des cas, cette méfiance s'étend aux intervenants sociaux (éducateurs, psychologues). II est clair que ces adolescents ont appris à compter sur eux-mêmes pour s'en sortir, mais, en contrepartie, cette force peut être associée à une méfiance à l'égard des adultes. Ainsi, Maxime dit : " II n'y a rien qui m'a aidé, c'est juste ma volonté. Il n'y a rien qui m'a aidé, il n'y a personne qui m'a aidé. Ils ne t'aident pas ici, ils te calent. » Ou Marcel qui affirme : «Ne pas faire trop confiance au monde, fais tes affaires par toi-même. Compter sur toi et ne pas tout le temps compter sur les autres. [...] Je ne trusterais pas full les adultes. »

Une grande perception de contrôle semble aussi, dans certains cas, entraîner une certaine vulnérabilité chez le jeune, particulièrement lorsque cette perception devient un frein à la demande d'aide. "Moi, je suis un gars qui veut tout contrôler mes affaires au complet. [...] Quand quelqu'un vient empiéter sur mes affaires, je vire fou. [...] Je suis autonome, puis je n'ai pas besoin de personne. » (Maxime) 


\section{Les caractéristiques environnementales}

Sur le plan des caractéristiques environnementales, une des zones de vulnérabilité chez ces jeunes est certainement le retour éventuel dans un milieu social où la déviance constitue souvent la norme. Par exemple, Maxime affirme : " Je garde mes chums. Je ne changerais pas de chums parce que j'étais un grand consommateur, puis là, je ne consomme plus. Mes chums vont rester les mêmes. Ils consommeront. Moi, si je ne veux pas consommer, je ne consommerai pas. »

Des relations problématiques avec un adulte significatif ou une absence de relation peuvent également constituer des facteurs de vulnérabilité pour le jeune. La plupart des jeunes cherchent, en effet, à établir des liens significatifs avec leurs parents, même lorsque ces derniers collaborent de façon minimale à l'établissement de ces liens.

Bien ça disait qu'il fallait qu'il s'occupe de moi, qu'il me prenne... Vraiment qu'il s'occupe de moi, qu'il m'appelle, qu'il fasse des trucs de même. Mais lui, il dit tout le temps : « t'as juste à m'appeler », mais il ne m'appelle jamais. (Fanny)

Enfin, certains jeunes sont aussi extrêmement isolés socialement. Ils savent, en effet, qu'ils ne pourront jamais vraiment compter sur les membres de leur entourage pour leur venir en aide. Ainsi, Marco dit ne pouvoir compter que sur le centre d'accueil: «parce que j'ai juste eux autres ».

\section{DISCUSSION}

La présente étude contribue à l'avancement des connaissances sur la résilience chez les jeunes hébergés en milieu substitut. Donner directement la parole aux jeunes en cours de placement a permis de mieux saisir leur propre compréhension de la situation. Cette approche apporte un point de vue complémentaire essentiel dans la recherche sur la résilience, puisqu'elle peut aider à dépasser certaines des limites occasionnées par la puissance explicative limitée des modèles quantitatifs.

Plus spécifiquement, cette recherche permet de saisir l'équilibre qui existe entre les facteurs de protection individuels et environnementaux. En effet, les jeunes résilients rencontrés nous ont parlé de leurs forces personnelles et également des facteurs de protection présents dans leur environnement. Ces résultats montrent clairement la nécessité d'avoir une vision multidimensionnelle du concept de résilience. Comme le soulignent Luthar, Cicchetti et Becker (2000), il existe encore une confusion conceptuelle dans ce champ, certains se centrant uniquement sur les attributs personnels en négligeant les aspects environnementaux qui sont associés à la résilience.

En ce qui concerne ces aspects environnementaux, la présente étude met en lumière la place primordiale qu'occupent des adultes significatifs dans la vie des jeunes placés, ce qui confirme les résultats obtenus par d'autres auteurs (Jackson et Martin, 1997; Lösel et Bliesener, 1994; Rutter et al., 1995). Nous avons vu notamment que le milieu 
familial, et particulièrement la mère, continue pour plusieurs jeunes d'être une source essentielle de soutien, et ce, malgré toutes les difficultés que ces jeunes ont pu vivre dans le passé. Même en contexte de placement, il ne faut donc pas négliger les facteurs de protection présents dans l'environnement naturel des jeunes.

Les résultats obtenus dans l'étude montrent aussi que ceux qui ont réussi à surmonter leurs épreuves ont pu compter sur la présence de personnes significatives extérieures à la famille immédiate et qui ont bien voulu leur servir, à un moment ou à un autre de leur vie, de « tuteur de remplacement »(Cyrulnik, 2003). À ce chapitre, le rôle joué par les intervenants est indéniable. D'ailleurs, lorsqu'on interroge des jeunes sur leur satisfaction à l'égard du placement, l'importance du lien avec les intervenants et l'apport de ces derniers dans leur réseau de soutien sont régulièrement mis en lumière (Brady et Caraway, 2002; Courtney et al., 2001). Cela dit, notre étude montre aussi une facette plus sombre de la situation. En effet, plusieurs jeunes ont mentionné éprouver du ressentiment, voire de la colère, envers certains intervenants et à l'égard du placement de façon plus générale.

Sur le plan individuel, les facteurs de protection qui ont été identifiés sont relatifs à l'estime de soi, à la confiance des jeunes vis-à-vis de leurs capacités de s'en sortir, aux stratégies d'adaptation qu'ils déploient ainsi qu'à la perception de contrôle sur leur vie. Ces éléments peuvent être mis en parallèle avec les facteurs individuels relevés dans la recension des écrits. Toutefois, la présente étude permet d'approfondir ces éléments en montrant notamment qu'ils peuvent comporter un revers. Par exemple, l'impression d'être en contrôle peut parfois s'accompagner du sentiment irréaliste de pouvoir tout contrôler; croire en ses capacités peut signifier aussi que l'on doit être entièrement autonome.

Cette étude montre également que les facteurs de protection individuels se construisent en interaction avec l'environnement. À cet égard, les jeunes ont insisté en particulier sur les expériences valorisantes qu'ils ont pu vivre et qui ont contribué à leur estime d'eux-mêmes. Les personnes qui les ont soutenus dans leur cheminement sont venues aussi alimenter cette confiance en leurs capacités de s'en sortir. II ne faut donc pas voir les jeunes résilients comme des héros inaccessibles possédant des attributs que les autres n'ont malheureusement pas, mais bien essayer de nourrir les forces individuelles de chaque jeune. Comme cette recherche le montre, malgré les graves difficultés auxquelles ils doivent faire face, les jeunes ont le potentiel nécessaire pour s'en sortir. Nous pouvons certes nous sentir impuissants devant l'ampleur et la complexité des problématiques présentées, et les mesures envisagées peuvent nous paraître bien modestes. Cependant, l'étude de la résilience montre que des événements possibles à l'intérieur des services de protection, que ce soit une rencontre significative ou l'implication dans une activité dans laquelle le jeune réussit, peuvent constituer des éléments porteurs d'espoir.

Notre étude comporte toutefois certaines limites. À ce sujet, il demeure nécessaire de rappeler, en terminant, que son objectif n'était pas de généraliser les résultats à l'ensemble de la population des jeunes hébergés en milieu substitut. Nous pouvons 
ajouter que la majorité des adolescents rencontrés dans le cadre de la présente étude vivaient en centre de réadaptation et que deux d'entre eux résidaient dans un autre type de ressource. Les conditions de vie et les problématiques de ces jeunes étant différentes, il serait souhaitable de mener des recherches distinguant mieux la portée de ces éléments contextuels dans le développement de la résilience (Rutter, 2000). II serait également utile de poursuivre le suivi de ces jeunes jusqu'à la fin de leur placement. Les conditions adverses présentes dans le milieu naturel ainsi que les difficultés personnelles de ces adolescents constituent des zones de vulnérabilité qui peuvent mettre en péril le processus amorcé.

Soulignons enfin que nous nous sommes appuyés sur une vision du concept de résilience impliquant un jugement social, normatif, basé sur la présence de compétences attendues chez des adolescents confrontés à de multiples risques. Cette approche ne rend pas compte du caractère subjectif et construit de la définition de la résilience. Des jeunes peuvent aussi se percevoir comme étant résilients et capables de surmonter les nombreux risques auxquels ils doivent faire face, sans pour autant être perçus comme résilients par les adultes qui prennent soin d'eux ou par la société en général (Ungar et Teram, 2000). Or, ces jeunes n'ont pas été entendus au cours de la présente étude.

\section{CONCLUSION}

Cette étude qualitative permet de mieux saisir l'expérience des jeunes placés en milieu substitut et leur interprétation des éléments qui ont joué un rôle dans leur trajectoire vers la résilience. Elle a mis en lumière les facteurs de protection, tant individuels qu'environnementaux, présents dans l'environnement des jeunes et également certaines zones de vulnérabilité qui peuvent faire dévier le jeune de cette trajectoire. Les résultats obtenus permettent d'envisager un certain nombre de cibles à atteindre afin de favoriser la résilience chez les jeunes pour qui le processus ne se met pas en place de façon naturelle. À cet égard, soulignons l'importance de miser sur les forces des jeunes; de soutenir leur perception d'avoir du contrôle sur ce qui leur arrive; de leur donner des occasions de participer à des activités valorisantes; de soutenir et de favoriser la création de liens significatifs avec les membres de la famille, les adultes non apparentés et bien sûr les intervenants. Cela dit, pour reprendre l'image de Werner (2005), mieux comprendre la résilience ne signifie pas que nous avons trouvé les ingrédients de la «potion magique » qui agit toujours avec tous de la même façon. II faut plus globalement viser la création d'environnements « attentifs » à la résilience, autrement dit de contextes propices à son émergence. Dans cette optique, non seulement le jeune doit-il être la cible du changement, mais aussi les adultes et les intervenants qui le côtoient, sans oublier bien sûr les environnements où ces personnes évoluent. Cette forme de pratique ne garantit certes pas le changement, mais elle en accroît la possibilité. 


\section{RÉFÉRENCES BIBLIOGRAPHIQUES}

BOYATZIS, R. (1998). Transforming Qualitative Information, Thousand Oaks, Sage Publications.

BRADY, L.K., et J.S. CARAWAY (2002). " Home away from home: Factors associated with current functioning in children living in a residential treatment setting », Child Abuse and Neglect, vol. 26, p. 1149-1163.

Cloutier, R. (2000). Familles d'accueil et intervention jeunesse. Analyse de la politique de placement en ressource de type familial, Québec, ministère de la Santé et des Services sociaux.

Courtney, M.E., I. Piliavin, A. Grogan-Kaylor et A. Nesmith (2001). « Foster youth transitions to adulthood: a longitudinal view of youth leaving care », Child Welfare, vol. 80, p. 685-717.

CYRULNIK, B. (2003). Le murmure des fantômes, Paris, Éditions Odile Jacob, 259 p.

FRASER, M.W., J. RICHMAN et M.J. GaLINSKY (1999). «Risk, protection, and resilience: Toward a conceptual framework for social work practice », Social Work Research, vol. 23, p. 131-143.

HENRY, D.L. (1999). «Resilience in maltreated children: Implications for special needs adoption », Child Welfare, vol. 78, $\mathrm{n}^{0}$ 5, p. 519-540.

JACKSON, S., et P.-Y. MARTIN (1998). "Surviving the care system: Education and resilience », Journal of Adolescence, vol. 21, p. 569-583.

LAPERRIĖRE, A. (1997). «Les critères de scientificité des méthodes qualitatives », dans J. Poupart, J.-P. Deslauriers, L.-H. Groulx, A. Laperrière, R. Mayer et A.P. Pires (dir.), La recherche qualitative, Montréal, Gaëtan Morin, p. 365-387.

LöSEL, F., et T. BLIESENER (1994). "Some high-risk adolescents do not develop conduct problems: A study of protective factors », International Journal of Behavior Development, vol. 17, p. 753-777.

LUTHAR, S.S. (1997). « Sociodemographic disadvantage and psychosocial adjustment: Perspectives from developmental psychopathology », dans S.S. LUTHAR, J.A. BURACK, D. CICCHETTI et J.R. WEISz (dir.), Developmental Psychopathology, Perspectives on Adjustment, Risk and Disorder, New York, Cambridge University Press, p. 459-485.

Luthar, S.S., D. CicchettI et B. BeCKer (2000). «The Construct of Resilience: A Critical Evaluation and Guidelines for Future Work », Child Development, vol. 71, n 3, p. 543-562.

MASTEN, A.S., et J.D. COATSWORTH (1998). « The development of competence in favorable and unfavorable environments, Lessons from research on successful children », American Psychologist, vol. 53, p. 205-220.

Mayer, R., F. Ouellet, M.-C. Saint-Jacques et D. Turcotte (2000). Méthodes de recherche en intervention sociale, Boucherville, Gaëtan Morin, 409 p.

Pauzé, R., J. Toupin, M. Déry et H. Mercier (2000). Projet FASS. Les soins aux jeunes en difficulté, synthèse du rapport, Québec, Centre jeunesse de Québec-Institut universitaire.

RUTTER, M. (2000). « Children in substitute care: Some conceptual considerations and research implications », Children and Youth Services Review, vol. 22, p. 685-703. 
RUTTER, M. (1990). "Psychosocial resilience and protective mechanisms », dans J. RolF, A.S. Masten, D. Cicchetti, K.H. Nuechterlein et S. Weintraum (dir.), Risk and Protective Factors in the Development of Psychopathology, New York, Cambridge University Press, p. 181-214.

RUTTER, M., et D. QUINTON (1984). « Long-term follow-up of women institutionalized in childhood: Factors promoting good functioning in adult life », British Journal of Developmental Psychology, vol. 2, p. 191-204.

RUTTER, M., D. QUINTON et J. HILL (1995). « Adult outcome of institution-reared children: Males and females compared », dans L. ROBINS et M. RUTTER (dir.), Straight and Devious Pathways from Childhood to Adulthood, New York, Cambridge University Press, p. 135-157.

UnGar, M., et E. Teram (2000). « Drifting toward Mental Health: High-Risk Adolescents and the Process of Empowerment », Youth and Society, vol. 32, $\mathrm{n}^{\circ}$ 2, p. 228-252.

WEINER, A., et H. KUPERMINTZ (2001). « Facing adulthood alone: the long-term impact of family break-up and infant institutions, a longitudinal study », British Journal of Social Work, vol. 31, $n^{0} 2$, p. 213-234.

WERNER, E.E. (2005). «What can we learn about resilience from large-scale longitudinal studies? », dans S. GoldSTEIN et R.B. BROokS (dir.), Handbook of Resilience in Children, New York, Kluwer Academic, p. 91-106. 